\title{
3-D HOS simulations of extreme waves in open seas
}

\author{
G. Ducrozet, F. Bonnefoy, D. Le Touzé, and P. Ferrant \\ Laboratoire de Mécanique des Fluides, Centrale Nantes, 1 rue de la Noë, 44300 Nantes, France
}

Received: 25 September 2006 - Revised: 8 January 2007 - Accepted: 16 January 2007 - Published: 25 January 2007

\begin{abstract}
In the present paper we propose a method for studying extreme-wave appearance based on the HigherOrder Spectral (HOS) technique proposed by West et al. (1987) and Dommermuth and Yue (1987). The enhanced HOS model we use is presented and validated on test cases. Investigations of freak-wave events appearing within longtime evolutions of 2-D and 3-D wavefields in open seas are then realized, and the results are discussed. Such events are obtained in our periodic-domain HOS model by using different kinds of configurations: either i) we impose an initial 3-D directional spectrum with the phases adjusted so as to form a focused forced event after a while, or ii) we let 2-D and 3-D wavefields defined by a directional wave spectrum evolve up to the natural appearance of freak waves. Finally, we investigate the influence of directionality on extreme wave events with an original study of the 3-D shape of the detected freak waves.
\end{abstract}

\section{Introduction}

In open seas, ships and marine structures are periodically exposed to extreme waves, which constitute a major problem for both structure integrity and human safety. These extreme physical events are both three-dimensional and highlynonlinear phenomena, making their numerical study challenging. Their occurrence may be related to a wave energy focusing which derives from a number of factors: wavecurrent interaction, bathymetry, wind effects, self-focusing instabilities, directional effects, etc. For a review on the different mechanisms of formation of such events, see Kharif and Pelinovsky (2003). In the present work, we mainly interest ourselves in long-time evolutions of wavefields in deep water or finite constant depth without wind or current.

Correspondence to: G. Ducrozet

(guillaume.ducrozet@ec-nantes.fr)
Historically, sea-spectra evolutions have been studied through weakly-nonlinear approaches where a limited number of wave-component interactions are accounted for. The first results derived using such a mode-coupling approach have been published in the early 1960s by Phillips (1960), Longuet-Higgins (1962) and Hasselmann (1962), but they were limited to the obtention of energy exchange rates resulting from the wave interactions. The full time evolution of the wave system has then been described by Zakharov (1968), who derived the mode rate equations. In their integral form (Zakharov, 1968) obtained using the Hamiltonian formalism, the latter equations have been solved using reduced expressions limited to four-wave or five-wave interactions. Nonetheless, it is only during the last two decades that these reduced Zakharov equations have been fully derived and solved (see e.g. Stiassnie and Shemer, 1984; Krasitskii, 1994; Annenkov and Shrira, 2001). Another direction that has been followed using also a limited order in wave steepness, has been to add the assumption of a narrow-banded wave spectrum. The weakly-nonlinear equations thus obtained have been widely used and enhanced since the first application of the Non-Linear Schrödinger equation to the gravity-wave hydrodynamics (in Zakharov, 1968). Among others (see Kharif and Pelinovsky, 2003, for a wider review), the derived equations include also the Davey-Stewardson system for a finite-depth formulation, the Dysthe (1979) equation which includes the fourth order in wave steepness, and modified versions of the latter to account for a broader bandwidth of wave spectrum (see e.g. Trulsen and Dysthe, 1996).

Within the framework of the latter weakly-nonlinear approaches, the extreme wave events referred to as "freak" or "rogue" waves have been studied as resulting from wave geometrical or spatio-temporal focusing, or from modulational Benjamin-Feir instabilities. However, since freak waves have large amplitudes, high steepness, and short duration, Kharif and Pelinovsky (2003) note that the assumptions of weak nonlinearity and narrow-banded

Published by Copernicus GmbH on behalf of the European Geosciences Union. 
spectrum are not fully valid. Moreover, the randomness of the 3-D wavefields involved attenuates the Benjamin-Feir instability phenomenon (see Kharif and Pelinovsky, 2003). The use of time-domain fully-nonlinear potential models should therefore be the best suitable approach to study such extreme events. Nonetheless, the numerical simulation of these fully-nonlinear open-sea evolutions is today still very challenging. The Boundary Element Method (BEM), classically employed within the latter class of methods, remains indeed too slow to reproduce square kilometers of ocean long-time evolution. Actually, the temporal and spatial scales involved, and the smallness of the highfrequency waves included in the sea spectrum lead to huge requirements in terms of space and time grids (typically millions of spatial nodes and thousands of time steps). An alternative approach is to use spectral resolutions based on the same equations derived by Zakharov (1968), but in their fully-nonlinear version before expressing them into wave-component interactions.

Such an approach has been proposed in 1987 respectively by West et al. (1987) and Dommermuth and Yue (1987); it is known as Higher-Order Spectral (HOS) method and permits the fully-nonlinear simulation of gravity-wave evolution within periodic unbounded 3-D domains. This method is also called Dirichlet-Neumann Operator (Craig and Sulem, 1993) which, in its accelerated version (Vijfvinkel, 1996), is equivalent to the HOS (cf. Le Touzé, 2003; Schäffer, 2005). With respect to classical time-domain models such as the BEM, this spectral approach presents the two assets of its fast convergence and its high computational efficiency (by means of FFTs), allowing to accurately simulate long-time 3-D seastate evolutions with fine meshes. A number of studies relative to open-sea evolutions have been carried out by using HOS, see e.g. Brandini (2001) or Brandini and Grilli (2001) for the apparition of natural freak waves in a 2-D spectrum, and the propagation of 2-D regular waves with 3-D modulational instabilities; or Tanaka (2001a) for a study of the nonlinear evolution of a 3-D spectrum.

In the present paper, we use a HOS model which is an enhanced version of the order-consistent HOS originally proposed (see West et al., 1987) which has been extensively validated. In this model, specific care has especially been paid to aliasing matters (see Bonnefoy, 2005) and numerical efficiency. This allows to accurately simulate on a singleprocessor PC thousands of wave spectral peak periods in 2$\mathrm{D}$, and the evolution during tens of periods of square kilometers of ocean in 3-D. Two kinds of simulations of extreme wave events appearing in open-sea evolutions are shown and discussed in the present work. First, we let a directional wave spectrum evolve, with phases initially artificially adjusted so as to obtain a forced focusing event after a while. Second, 2$\mathrm{D}$ and 3-D spectra with random initial phases are considered and the occurrence of natural freak waves within their evolutions is investigated and discussed. Finally, the influence of the directionality on the freak waves formation is investigated. A double zero-crossing analysis of the numerical wavefield allows us to define two characteristic lengthes, in the mean and in the transverse directions of propagation. A parametric study is then run on the directional spreading, and results are discussed in terms of freak waves height, transverse extension, frequency of formation, and groupiness.

\section{Formulation and validation}

\subsection{Hypothesis and general equations}

We consider an open periodic fluid domain $D$ representing a rectangular part of the ocean of infinite depth and dimensions $\left(L_{x}, L_{y}\right)$. We choose a cartesian coordinate system with the origin $O$ located at one corner of the domain $D$. The $O_{z}$ axis is vertical and oriented upwards, and the level $z=0$ corresponds to the mean water level. The notation $\mathbf{x}$ stands for the $(x, y)$ vector.

The fluid simulated is water, which is assumed to be incompressible and inviscid. The flow is also considered irrotational. With these assumptions one can apply potential flow theory, the velocity $\mathbf{V}$ derives from a potential $\mathbf{V}(\mathbf{x}, z, t)=\tilde{\mathbf{\nabla}} \phi(\mathbf{x}, z, t), \tilde{\mathbf{\nabla}}$ representing the gradient and $\phi$ the velocity potential. Then, continuity equation $(\operatorname{div} \mathbf{V}=0)$ becomes the Laplace's equation

$$
\Delta \phi=0 \quad \text { in } D
$$

Following Zakharov (1968), the fully-nonlinear free surface boundary conditions can be written in terms of surface quantities, namely the single-valued free surface elevation $\eta(\mathbf{x}, t)$ and the surface potential $\phi^{s}(\mathbf{x}, t)=\phi(\mathbf{x}, \eta, t)$

$$
\begin{aligned}
\frac{\partial \phi^{s}}{\partial t} & =-g \eta-\frac{1}{2}\left|\nabla \phi^{s}\right|^{2}+\frac{1}{2}\left(1+|\nabla \eta|^{2}\right)\left(\frac{\partial \phi}{\partial z}\right)^{2} \\
\frac{\partial \eta}{\partial t} & =\left(1+|\nabla \eta|^{2}\right) \frac{\partial \phi}{\partial z}-\nabla \phi^{s} \cdot \nabla \eta
\end{aligned}
$$

on $z=\eta(\mathbf{x}, t)$. This way, the only remaining non-surface quantity is the vertical velocity $\frac{\partial \phi}{\partial z}$ which will be evaluated thanks to the West et al. (1987) order-consistent HighOrder Spectral scheme. The two surface quantities are then marched in time using an efficient 4th-order Runge-Kutta scheme featuring an adaptative step-size control and in which the linear part of the equations is integrated analytically.

\subsection{Boundary conditions and spectral basis functions}

In the previous section the free surface boundary conditions have been established. It remains to express the conditions on the sides, and the bottom of the domain if there is one. The considered domain $D$ is periodic in both the $x$ and $y$ directions. This is expressed by the following two conditions

$$
\begin{aligned}
& (\phi ; \eta)(x=0, y, z, t)=(\phi ; \eta)\left(x=L_{x}, y, z, t\right) \\
& (\phi ; \eta)(x, y=0, z, t)=(\phi ; \eta)\left(x, y=L_{y}, z, t\right)
\end{aligned}
$$


In the domain $D$ defined, the following basis functions $\psi_{m n}^{f g}$ individually satisfy the set of Eqs. $(1,4,5)$

$\psi_{m n}^{f g}=f\left(k_{m} x\right) g\left(k_{n} y\right) \exp \left(k_{m n} z\right)$

where $f$ and $g$ can either be the sine or the cosine functions, and $k_{m}=m \pi / L_{x}, k_{n}=n \pi / L_{y}$ and $k_{m n}=\sqrt{k_{m}^{2}+k_{n}^{2}}$ the wavenumbers associated with mode $(m, n)$. The velocity potential $\phi$ can then be expressed on this basis as

$$
\phi(\mathbf{x}, z, t)=\sum_{m=0}^{+\infty} \sum_{n=0}^{+\infty} A_{m n}^{f g}(t) f\left(k_{m} x\right) g\left(k_{n} y\right) \exp \left(k_{m n} z\right)
$$

This way, the velocity potential is fully determined by the knowledge of the time-dependent coefficients $A_{m n}^{f g}(t)$.

\subsection{The Higher-Order Spectral method}

In the free surface conditions $(2,3)$ which are marched in time to get the evolution of $\eta$ and $\phi^{s}$, the only remaining unknown is the vertical velocity $\frac{\partial \phi}{\partial z}$. The HOS method relies first on the expansion of the velocity potential located at the exact free surface position, into series of complementary unknown quantities and Taylor series about the mean water level. From these quantities a fast-converging iterative process is employed to get the vertical derivative $\frac{\partial \phi}{\partial z}$ at the exact free surface position. In this process, it should be kept in mind that the HOS technique retains the fully-nonlinear feature of the solution. Indeed, the evaluation of $\frac{\partial \phi}{\partial z}$ is an inner process which is not correlated to the fully-nonlinear solution of the problem; the fully-nonlinear free surface boundary conditions are actually solved at the exact free surface position. For further details about the procedure see Appendix A.

The HOS formulation allows us to use a very efficient FFT-based solution scheme with numerical cost growing as $N \log _{2} N, N$ being the number of modes. Moreover, an acceleration of the solution process has been implemented, see Appendix B. It is also to notice that the free-surface boundary conditions (Eqs. 2 and 3) and the HOS iterative process contain nonlinear products that have to be carefully treated. These products are computed in the physical space and are dealiased cautiously, see Appendix C.

\subsection{Validation}

Various validations of the HOS formulation have been performed, see in particular the original article by West et al. (1987). To further validate the model we use, all the stability and accuracy tests found in the literature have been redone, confirming both the efficiency and the accuracy of the present implementation where specific care has been paid to the dealiasing in particular (Le Touzé, 2003; Bonnefoy, 2005, Appendix C,). The model has also been verified on long-time evolutions 3-D cases. For instance, the simulations performed in Tanaka (2001a) or Tanaka (2001b) have
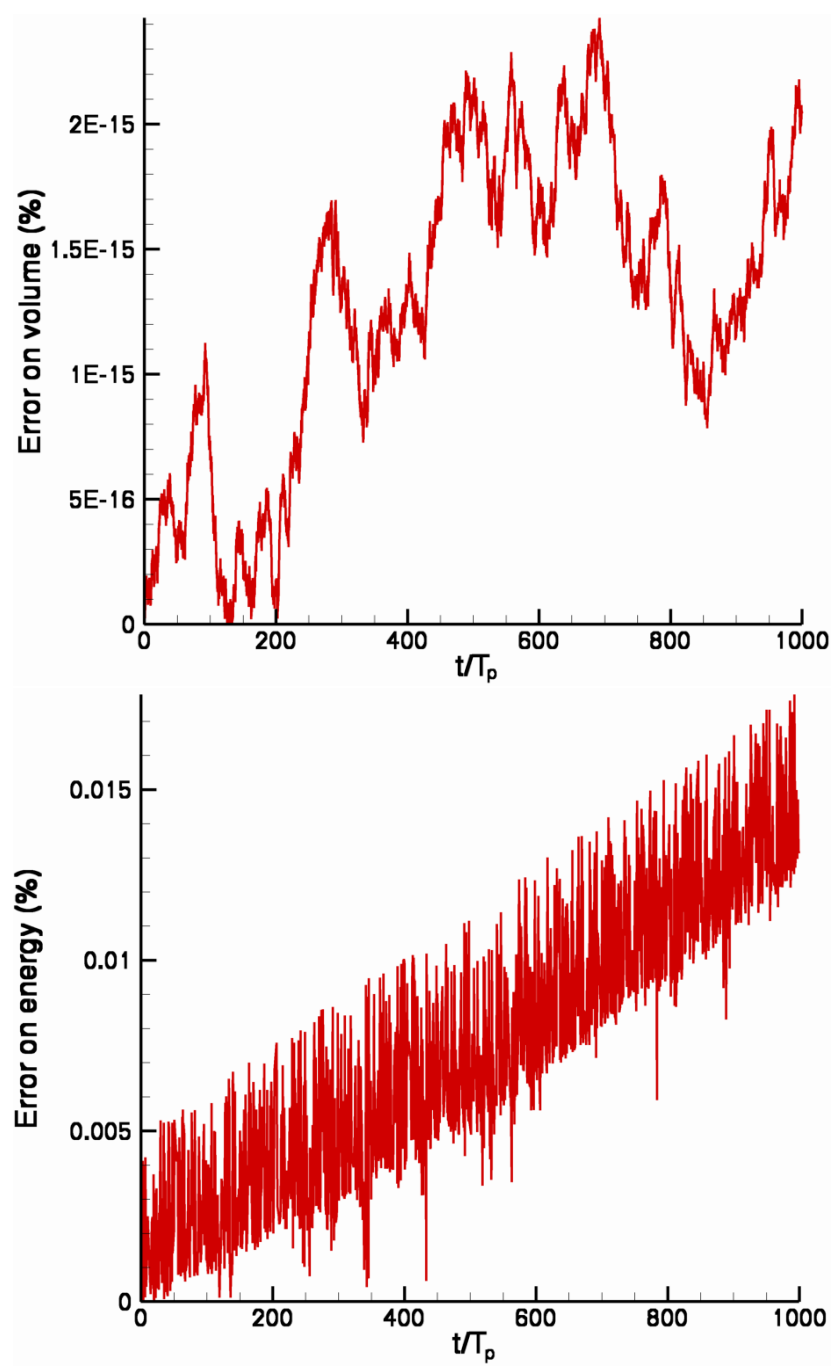

Fig. 1. Error on the volume (top) and energy (bottom) of the wavefield after 1000 peak periods of propagation $\left(M=8, N_{x}=2048\right.$ modes).

been re-run with very similar results on the evolution of 3-Dwave spectra in huge domains of computation. Such largescale numerical simulations with the HOS method permit the verification of Hasselmann's theory for the nonlinear energy transfer among surface gravity waves (Hasselmann, 1962). This nonlinear energy transfer is evaluated from the rate of change of different spectra and is successfully compared to the Hasselmann's theory.

In Fig. 1 is represented the evolution of the volume and the energy (kinematic plus potential energies of the fluid) during a 2-D simulation lasting 1000 wave spectral peak periods (see Sect. 3.2.1).

As expected since each of the basis functions used is exactly satisfying mass conservation, one can easily observe that the volume is conserved within the computer proces- 
Table 1. Error on the energy and CPU time per time step, as functions of the number of modes.

\begin{tabular}{cccc}
\hline$N_{x} \times N_{y}$ & $\begin{array}{c}\text { Error } \epsilon_{1} \\
\text { in } \%\end{array}$ & $\begin{array}{c}\text { Error } \epsilon_{2} \\
\text { in } \%\end{array}$ & $\begin{array}{c}\text { CPU time } \\
\text { in s }\end{array}$ \\
\hline $32 \times 16$ & 100 & 0.05 & 0.02 \\
$64 \times 32$ & 100 & 0.05 & 0.04 \\
$128 \times 64$ & 94 & 0.10 & 0.32 \\
$256 \times 128$ & 45 & 0.17 & 1.45 \\
$512 \times 256$ & 14 & 0.07 & 13 \\
$1024 \times 512$ & 4 & 0.005 & 65 \\
$2048 \times 1024$ & 2 & 0.002 & 410 \\
\hline
\end{tabular}

sor accuracy. Energy is also conserved with good accuracy, within less than $1.510^{-4}$ relative error after 1000 peak periods of propagation.

A study of convergence on 3-D irregular wavefields is more difficult to set up. Theoretically speaking though, the form of the basis functions in the spectral expansion leads to the same accuracy levels and convergence rates in both directions $(x$ and $y$ ) than in 2-D, which is easily verified in practice on simple cases. Thus, to assess the convergence of our HOS model, we refer to the several studies done in the literature (see in particular West et al., 1987; Dommermuth and Yue, 1987) on regular wavefields. The behavior of the present model on regular waves has also been assessed in detail for various steepness conditions (see Le Touzé, 2003). When simulating a directional wavefield, this verification is less easily done. Indeed, the random choice of initial phases leads to different simulations if we change the number of modes used in the calculations. Nonetheless, looking at global parameters of the wavefield, such as its energy, one can get an idea of the convergence of the results with respect to the number of modes. We distinguish here two kinds of errors on the energy, $\epsilon_{1}$ and $\epsilon_{2}$. $\epsilon_{1}$ represents the error due to the discretization of the initial spectrum. It is obtained by comparing the numerical energy at $t=0$ to the energy $E$ of the target wavefield, the latter being evaluated from the input spectrum (see Sect. 2.5). And $\epsilon_{2}$ is the error on the energy balance during the simulation (cf. Fig. 1). It is evaluated as the error between the numerical energy at $t=0$ and the same energy after 50 peak periods of propagation. In Table 1 is also reported the corresponding CPU time per time step on a $3 \mathrm{GHz}$-Xeon single-processor PC. Simulations are performed with $M=3$ and are fully dealiased.

The convergence of the computations with respect to the number of modes is first assessed in terms of initial condition. Looking at $\epsilon_{1}$ clearly shows that the initial wave energy converges toward the specified energy $E$ as we increase the number of modes in both directions. Second, the evolution of $\epsilon_{2}$ reveals that the numerical conservation of the energy is also rapidly improved with the increase of the number of

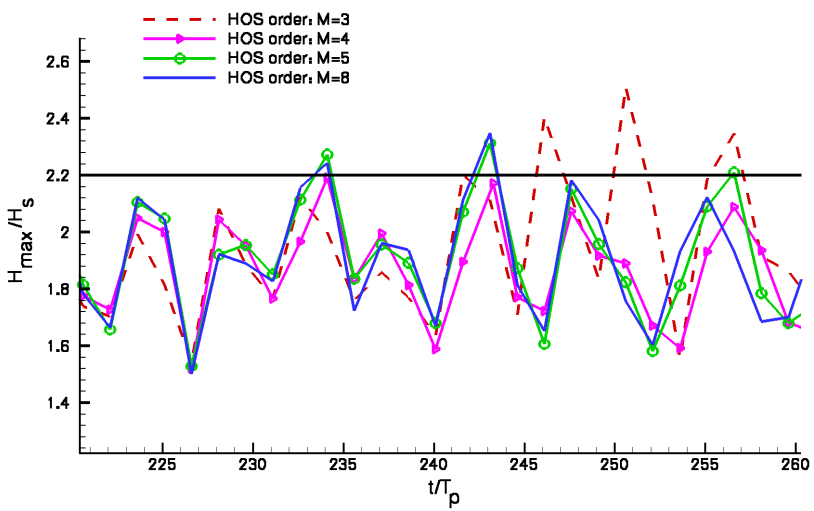

Fig. 2. Influence of HOS order M.

modes in the domain, once a sufficient number of modes has been used to capture the main structures present in the domain. Regarding the CPU time, the efficiency is pointed out with a rise comparable to $\mathrm{N} \log _{2} N$ ( $N$ being the total number of modes $N_{x} \times N_{y}$ ), as expected with the use of FFTs in the numerical scheme.

In Fig. 2, still on the same simulation case, is represented the influence of the HOS order $M$, which corresponds to the number of iterations chosen in the iterative process. In this figure is plotted the evolution of a parameter of interest, $H_{\max } / H_{S}$ (see Appendix D) for four different HOS orders $(M=3,4,5$ and 8$)$.

If one focuses on the freak wave event $\left(H_{\max } / H_{s}>2.2\right.$, see Appendix D) occurring after about 240 peak periods of propagation, one can notice that even after such long-time of simulation, the results are really close. However, the calculation with $M=3$ differs from the three others. Indeed, $M=3$ predicts a freak wave event at $t=250 T_{p}$ which is overestimated with respect to the calculations with $M=4,5$ and 8 . Further in detail, the computation with $M=4$ underestimates a bit the extreme event at $t=243 T_{p}$ when compared to the $M=5$ and 8 curves. The latter two results are almost superimposed, acknowledging the convergence of our calculation with $M$. Thus, the calculations will be performed with at least $M=5$ in the next sections.

\subsection{Initialization}

It has been demonstrated (Dommermuth, 2000) that the definition of adequate initial fields to start the computations is not straightforward, and can lead to numerical instabilities if done in an uncorrect way. Nonetheless, Dommermuth indicates that simulations can be initialized in a linear way; in this case a transition period (lasting from 5 to 10 peak periods of propagation) exists. After this establishment delay, the initial wavefield becomes a "realistic" fully-nonlinear one. In the simulations performed in the present work, which are long-time evolutions (see Sect. 3.2.1 and 3.2.2) of wave- 
fields, this transition period is taken into account through the use of a relaxation scheme allowing the use of such linear initial conditions. The free surface boundary conditions that can be classically written in the following form

$$
\frac{\partial \phi^{s}}{\partial t}+g \eta=F, \quad \frac{\partial \eta}{\partial t}-W^{(1)}=G
$$

are modified following the Dommermuth (2000) adjustment scheme of the nonlinear terms as follows

$$
\begin{aligned}
\frac{\partial \phi^{s}}{\partial t}+g \eta & =F\left(1-\exp \left[-\left(\frac{t}{T_{a}}\right)^{n}\right]\right) \\
\frac{\partial \eta}{\partial t}-W^{(1)} & =G\left(1-\exp \left[-\left(\frac{t}{T_{a}}\right)^{n}\right]\right)
\end{aligned}
$$

We chose to set the two parameters as $T_{a}=10 T_{p}$ and $n=4$, still following Dommermuth (2000).

We have chosen to take the same kind of initial wavefields as in Tanaka (2001a). These wavefields are defined by a directional spectrum, typically JONSWAP or PiersonMoskowitz (P-M) in deep water. In his paper, Tanaka found a relation between the so-called complex amplitude $b(\mathbf{k}, t)$ introduced by Zakharov (1968) and the directional spectrum $\Phi(\omega, \theta)$. The complex amplitude can then be related to the surface elevation $\eta$ and the surface velocity potential $\phi^{s}$

$b(\mathbf{k}, t)=\sqrt{\frac{\omega(\mathbf{k})}{2 k}} \hat{\eta}(\mathbf{k}, t)+i \sqrt{\frac{k}{2 \omega(\mathbf{k})}} \hat{\phi}(\mathbf{k}, t)$

with the hat denoting the Fourier transform and

$\omega(\mathbf{k})=\sqrt{g k} \quad$ and $k=|\mathbf{k}|$

$\eta$ and $\phi^{s}$ can also be related to $b$ with the following equations

$$
\begin{gathered}
\eta(\mathbf{x}, t)=\frac{1}{2 \pi} \int \sqrt{\frac{k}{2 \omega(\mathbf{k})}}\left[b(\mathbf{k}, t)+b^{*}(-\mathbf{k}, t)\right] e^{i \mathbf{k} \cdot \mathbf{x}} d \mathbf{k}(9) \\
\phi^{s}(\mathbf{x}, t)=\frac{1}{2 \pi} \int \sqrt{\frac{\omega(\mathbf{k})}{2 k}}\left[b(\mathbf{k}, t)-b^{*}(-\mathbf{k}, t)\right] e^{i \mathbf{k} \cdot \mathbf{x}} d \mathbf{k}(10)
\end{gathered}
$$

The relation between $b_{k}$ (discrete values of $\left.b(\mathbf{k}, t)\right)$ and $\Phi(\omega, \theta)$ is

$$
\left|b_{k}\right|=\frac{g^{2}}{2 \omega_{k}^{4}} \Phi(\omega, \theta) \delta k_{x} \delta k_{y}
$$

Therefore, we calculate the norm $\left|b_{k}(t=0)\right|$ with the previous Eq. (11) for the desired directional spectrum $\Phi(\omega, \theta)$ and the phase of $b_{k}(t=0)$ is determined by a random number in $[0,2 \pi]$. Then, we have constructed the initial wavefield of interest using Eqs. (9) and (10).

We classically define the directional spectrum $\Phi(\omega, \theta)$ as

$\Phi(\omega, \theta)=\psi(\omega) \times G(\theta)$

The spectrum is typically

$\psi(\omega)=\alpha g^{2} \omega^{-5} \exp \left(-\frac{5}{4}\left(\frac{\omega}{\omega_{p}}\right)^{-4}\right) \gamma^{\exp \left[-\frac{\left(\omega-\omega_{p}\right)^{2}}{2 \sigma^{2} \omega_{p}^{2}}\right]}$ with $\alpha$ being the Phillips constant and $\omega_{p}$ the angular frequency at the peak of the spectrum. The directionality is defined by

$G(\theta)= \begin{cases}A_{n} \cos ^{n} \theta, & |\theta| \leq \frac{\pi}{2} \\ 0, & |\theta|>\frac{\pi}{2}\end{cases}$

with,

$A_{n}= \begin{cases}\frac{\left(2^{p} !\right)^{2}}{\pi(2 p) !}, & \text { if } n=2 p \\ \frac{(2 p+1) !}{2\left(2^{p} p !\right)^{2}}, & \text { if } n=2 p+1\end{cases}$

Space and time are then normalized such that $\omega_{p}$ and $g$ are both equal to 1 . Thus, $\Phi(\omega, \theta)$ is the JONSWAP spectrum when

$\alpha=3.279 E, \quad \gamma=3.3, \quad \sigma=\left\{\begin{array}{c}0.07(\omega<1), \\ 0.09(\omega \geq 1)\end{array}\right.$

where $E$ is the dimensionless energy density of the wavefield. The significant wave height could be estimated by $H_{S} \approx 4 \sqrt{E}$ (Tanaka (2001b)).

\section{Freak wave formation}

Using the numerical model described in the previous sections, we study the formation of freak waves under two different approaches. First, the focusing of a directional spectrum is considered; it represents a common approach used in experimental investigations of the influence of these events on ships or marine structures. Second, the model is used to detect natural freak waves during long-time nonlinear simulations, both in two and three dimensions.

\subsection{Directional focusing}

In this section we study the formation of extreme events due to directional focusing. This artificial generation of freak waves is based on the linear focusing of directional components at given time and location in the domain (to compare with, the next section presents natural formation of extreme events during the propagation of the wavefield).

\subsubsection{Initial condition}

The initial wave pattern is described by a directional JONSWAP spectrum. The spectral components have their phase adjusted so that a large amount of energy is located in the middle of the fluid domain at a given time.

This adjustment is made through the choice of the initial phases of $b_{k}(t=0)$ (cf. Sect. 2.5). For a classical uniform 


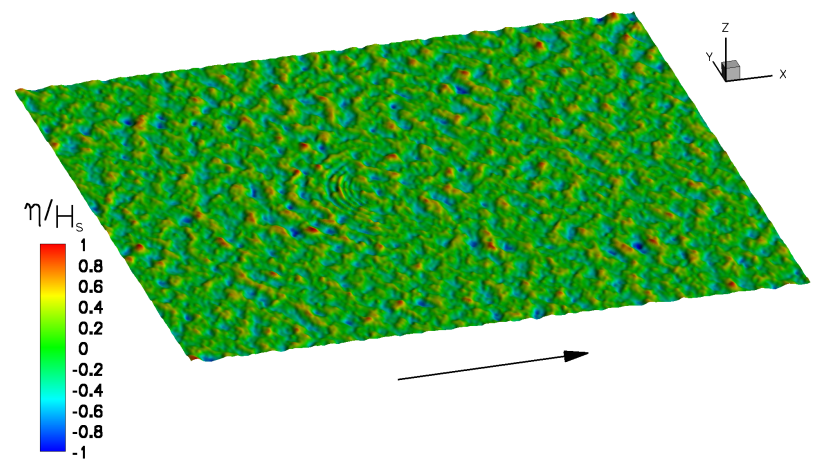

Fig. 3. 3-D initial surface elevation: $E=0.005$, $L_{x}=21 \lambda_{p} \times L_{y}=21 \lambda_{p}, \quad N_{x}=512 \times N_{y}=256$, HOS order $M=5$ and linear backward propagation during $20 T_{p}$.

irregular wave train, one has to choose a random number in $[0,2 \pi]$. By reducing this interval, for instance to

$$
\left[0,2 \pi\left(1-\frac{\alpha}{100}\right)\right]
$$

part of the energy is accumulated at $\mathbf{x}=\mathbf{0}$, which can be chosen as the middle of the domain we simulate.

Once this done, we perform a linear backward propagation during $T_{b}=20 T_{p}$. That is to say, each wave component $(m, n)$ is propagated at its own angular frequency $\omega_{m n}=\sqrt{g k_{m n}}$ during the backward propagation duration $T_{b}$. Eq. (7) then becomes

$$
\begin{array}{r}
\phi\left(\mathbf{x}, z, t-T_{b}\right)=\sum_{m=0}^{+\infty} \sum_{n=0}^{+\infty} A_{m n}^{f g}(t) f\left(k_{m} x-\omega_{m n} T_{b}\right) \\
g\left(k_{n} y-\omega_{m n} T_{b}\right) \exp \left(k_{m n} z\right)
\end{array}
$$

The basis functions $f$ and $g$ being either sine or cosine functions.

We thus obtain an initial wavefield that we let further evolve. Figure 3 shows a view of the initial wavefield, with the arrow indicating the direction of propagation.

The focusing components create a concentric pattern observable in the center part of the domain. The numerical conditions are:

- Wave field characterized by $E=0.005$ i.e. $\alpha=0.016, H_{s}=0.28$ in non-dimensional quantities (with respect to $g$ and $\omega_{p}$ ),

- Domain length: $L_{x}=21 \lambda_{p} \times L_{y}=21 \lambda_{p}$,

- Number of modes used: $N_{x}=512 \times N_{y}=256$, HOS or$\operatorname{der} M=5$,

- Dimensional quantities give, if we fix $T_{p}=9.5 \mathrm{~s}$ (typical in North Sea): $\lambda_{p}=70 \mathrm{~m}$, dimensional domain area: $2870 \mathrm{~m} \times 2870 \mathrm{~m}$.

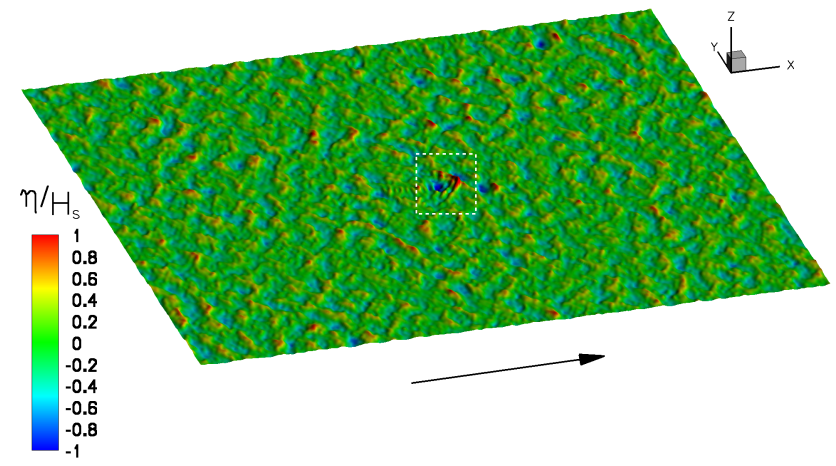

Fig. 4. 3-D surface elevation at $t=20.3 T_{p}$.

We use here classical wave spectrum parameters for North Atlantic. In particular, $E=0.005$ can be deduced from the well known scatter diagram of $T_{p}$ and $H_{s}$ in Haver et al. (2002) with pooled data from the Northern North Sea (about 70000 data points in the period 1973-2001). As explained in Socquet-Juglard (2005), the curve representing mean steepness $s$ equal to 0.1 in this diagram includes almost all data points and can be chosen as a good parameter for the description of highest wavefields in North Sea.

This mean steepness is defined as

$s=k_{p} \bar{a}=\frac{\sqrt{2} \pi^{2}}{g} \frac{H_{s}}{T_{p}^{2}}$

with $\bar{a}$ being the rms value of the amplitude. Using $H_{s}=4 \sqrt{E}$, one gets the following relation between $E$ and $s$

$E=\frac{s^{2} g^{2} T_{p}^{4}}{2(2 \pi)^{4}}=\frac{s^{2} g^{2}}{2 \omega_{p}^{4}}$

Thus, $s=0.1$ gives a dimensionless energy $E=0.005$.

\subsubsection{Results}

Figure 4 presents the 3 -D surface elevation at $t=20.3 T_{p}$ where the nonlinear elevation is maximum. The major part of the domain consists of unfocused components, while at the center the focused ones are superimposed and form the extreme event.

A closer view of the center of the domain is given in Fig. 5. The center of the latter figure is the linear focusing point which is defined as the location of the focusing for linear waves propagation (i.e. the location of focused waves before the linear backward propagation). The contour levels have been adjusted so that the shape of the wave appears more clearly. In this case, the focused wave consists of a steep front wave with a V-shaped crest, followed by a deep trough. The strongest focusing occurs after the linear focusing point, both in time ( $\left.t=20.3 T_{p}\right)$ and in space (see Fig. 5), as is often observed in focusing experiments (the maximum wave 


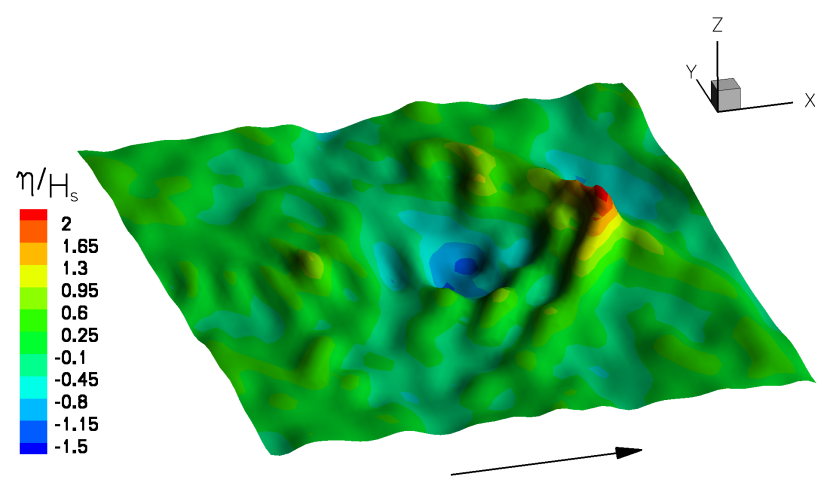

Fig. 5. Zoom of Fig. $4, t=20.3 T_{p}$.

elevation at the focusing point also occurs before the linear focusing time $t=20 T_{p}$ due to amplitude dispersion. The extreme wave generated this way reaches $H_{\max } / H_{s}>3.5$. The probability to have such event is really low and does not seem realistic (compared to known measurements in ocean). Therefore, the choice of the focused components of the spectrum has to be adapted to generate realistic waves. We then choose a more convenient approach to study the extreme events, looking for natural generation.

\subsection{Natural emergence}

In this section, we define an initial sea-state (as seen in Sect. 2.5) with typical natural parameters including random phases. Then, we let the sea-state evolve during a long-time of propagation and analyse the results looking for the extreme events that may appear in the domain. We show in this section only some of the behaviors observed in the conducted simulations. A larger number of simulations should be realized for a stochastically correct approach.

\subsubsection{Sample 2-D long-time evolution}

The first simulations are conducted in 2-D (i.e. without any directionality). The numerical conditions are:

- Wave field characterized by $E=0.0032$ i.e. $\alpha=0.011, H_{s}=0.23$ in adimensional quantities,

- Domain length: $L_{x}=82 \lambda_{p}$,

- Number of modes used: $N_{x}=2048$, HOS order $M=8$,

- Dimensional quantities give, if we fix $T_{p}=9.5 \mathrm{~s}$ (typical in North Sea): $H_{s}=5.1 \mathrm{~m}$ and $\lambda_{p}=140 \mathrm{~m}$. Dimensional domain length: $11480 \mathrm{~m}$.

Here is chosen $s=0.08$ (i.e. $E=0.0032$ ) which belongs to the range of classical values for North Sea wavefields (see Socquet-Juglard, 2005). It represents a typical steep wavefield, yet without being among the steepest ones.

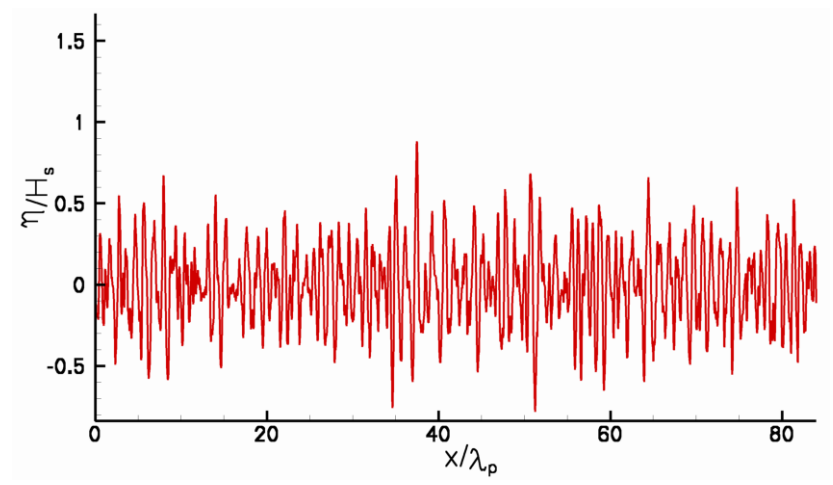

Fig. 6. 2-D initial surface elevation.

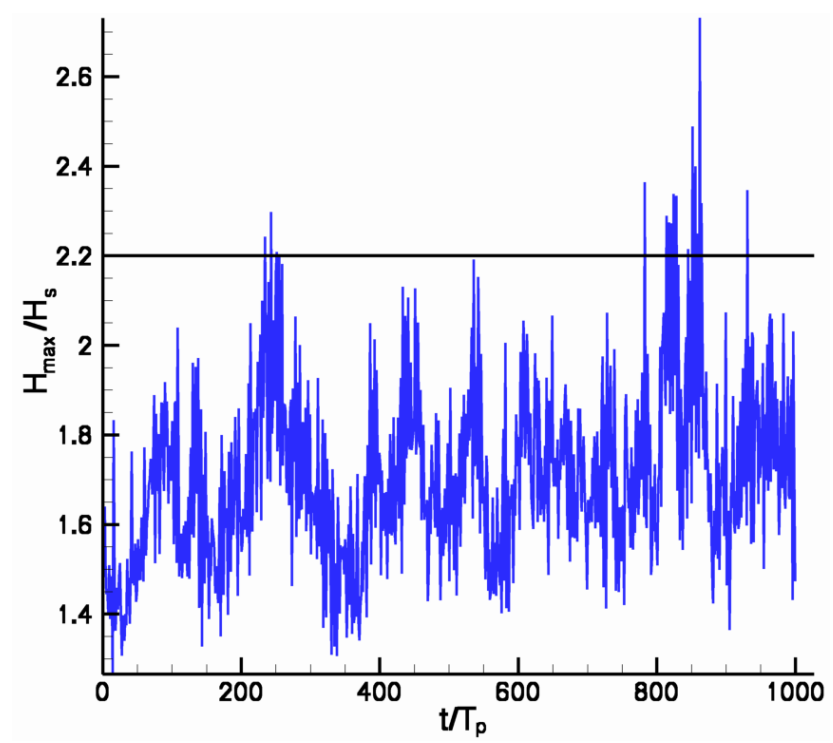

Fig. 7. Evolution of $H_{\max } / H_{S}$ during simulation.

In Fig. 6 is represented the initial surface elevation in the computational domain; waves will propagate from left to right at $t>0$. An analysis of this free surface elevation confirm that the significant wave height is, as expected, equal to 0.23 . We have then an irregular 2-D wavefield that we let evolve during 1000 peak periods. Dimensionally speaking, it corresponds to a simulation lasting for about $2 \mathrm{~h}$ and $40 \mathrm{~min}$ real time, within the huge $11.5 \mathrm{~km}$-long domain.

During the simulation, we look at the evolution of the parameter $H_{\max } / H_{s}$ which is suitable to characterize a so-called freak wave event when $H_{\max } / H_{s}>2.2$ (see Appendix D).

Figure 7 plots the evolution of this parameter against time. Several patterns can be observed all along the 1000 peak periods of simulation. Very brief events are detected at $t / T_{p}=800$ and 950 for example. Longer events are also present, i.e. that stay longer in the wavefield (see e.g. be- 


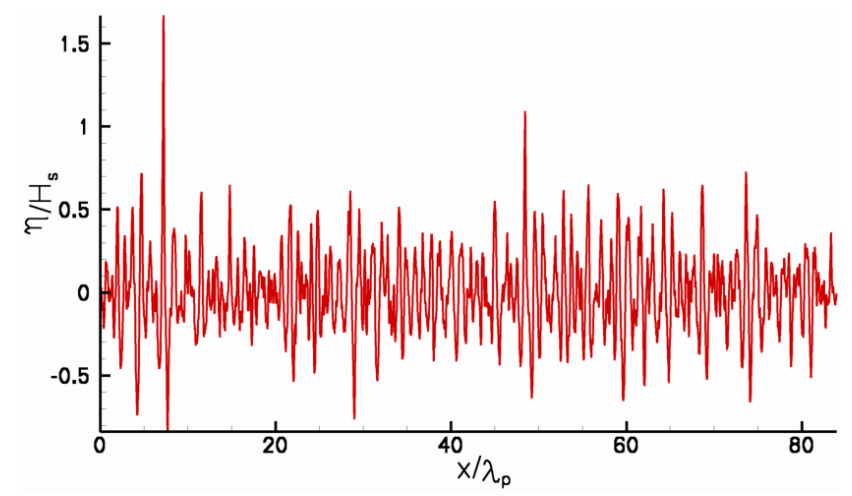

Fig. 8. 2-D surface elevation of the freak wave event: $t=860 T_{p}$.

tween $t / T_{p}=800$ and 900). The latter case corresponds to a high amplitude wavegroup that remains coherent for several periods in a row and produces successive extreme events. This second pattern is similar to the one described by Trulsen (2000) in its investigation to recreate the Draupner wave. As far as marine structures and ships are concerned, the probability of encountering such series of large waves is much greater than that of single large waves since the life time of the former is longer.

To further investigate this kind of wavegroup, we focus our attention on the series around $t=860 T_{p}$ in Fig. 7. Figure 8 represents the free surface elevation at $t=860 T_{p}$ when the highest wave of the series is produced. The extreme event consists of a single wave twice as high as the second highest wave in the domain.

One can observe on the closer view of the extreme event given in Fig. 9, a rather deep trough preceding the extreme wave. This corresponds to a wave height $H_{\max }=2.4 H_{s}$ and a steepness of $7 \%$ if evaluated as $H_{\max } / \lambda_{z c}$ with the measured zero-crossing wavelength $\lambda_{z c}=1.2$. This naturally emerged event fits well with the numerous reported observations of steep walls of water. I notice here that Brandini (2001) did same kind of simulations with a classical HOS method and perform statistical analysis of freakwave occurences. His long-time simulations are computed during $200 T_{p}$ on a domain of length $32 \lambda_{p}$ with a HOS order $M=4$. We produce here results on a longer time of simulations with a larger domain and a non-neglictable increase of HOS order (up to $M=8$ ).

However this simulation is two dimensional and one has to wonder about the effect of directionality on the occurrence of extreme events.

\subsubsection{Sample 3-D long-time evolution}

We study here the evolution of a 3-D directional wavefield. The wavefield and chosen numerical conditions are the same

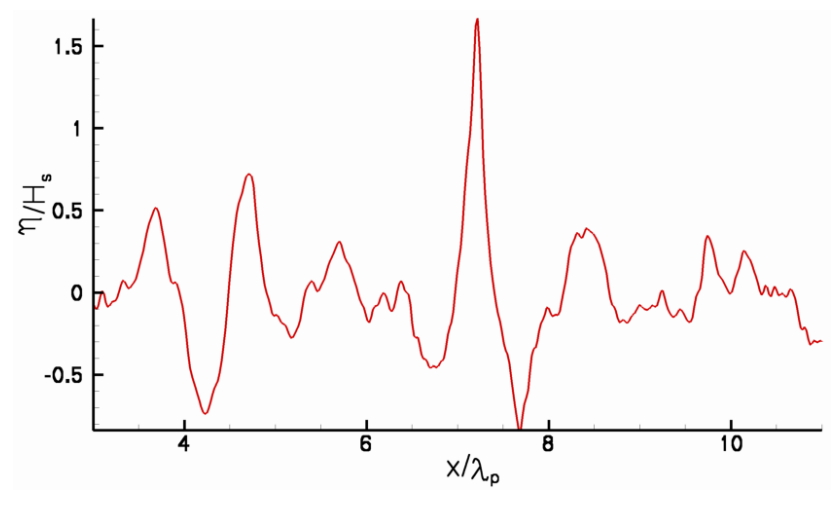

Fig. 9. Zoom on the 2-D freak wave event: $t=860 T_{p}$.

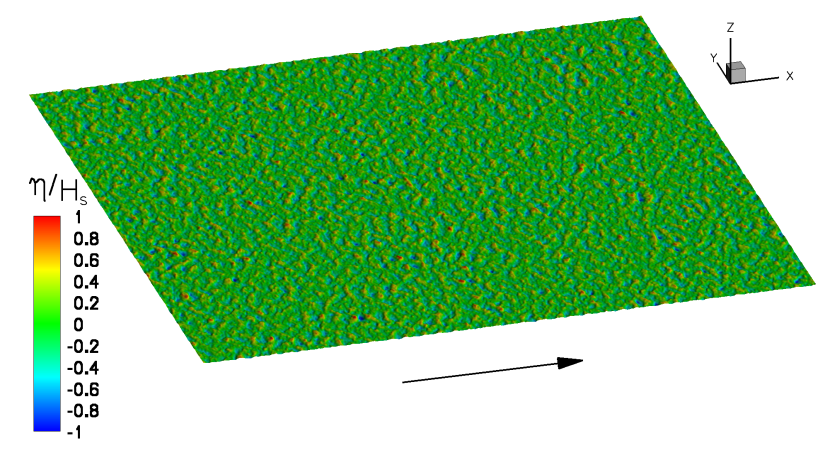

Fig. 10. 3-D initial surface elevation.

than before, except that we choose a mean steepness of the wavefield $s=0.1$ accounting for the highest sea-states:

- Wave field characterized by $E=0.005$ i.e. $\alpha=0.016, H_{s}=0.28$ in adimensional quantities,

- Domain length: $L_{x}=42 \lambda_{p} \times L_{y}=42 \lambda_{p}$,

- Number of modes used: $N_{x}=1024 \times N_{y}=512$, HOS order $M=5$,

- Dimensional quantities give, if we fix $T_{p}=9.5 \mathrm{~s}$ (typical in North Sea): $H_{s}=6.2 m$ and $\lambda_{p}=140 m$. Dimensional domain area: $5740 \mathrm{~m} \times 5740 \mathrm{~m}$.

The domain length in $x$ and the HOS order chosen have been slightly reduced compared to the previous 2-D case to keep a reasonable CPU time. With these numerical conditions, a simulation lasting 250 peak periods requires about $10 \mathrm{CPU}$ days on a $3 \mathrm{GHz}$-Xeon single-processor PC.

Figure 10 shows the initial surface elevation for a directionality parameter $n=2$ (cf. Eq. 12).

The simulation is run for 250 peak periods and the wave height is monitored to detect freak wave events (see 


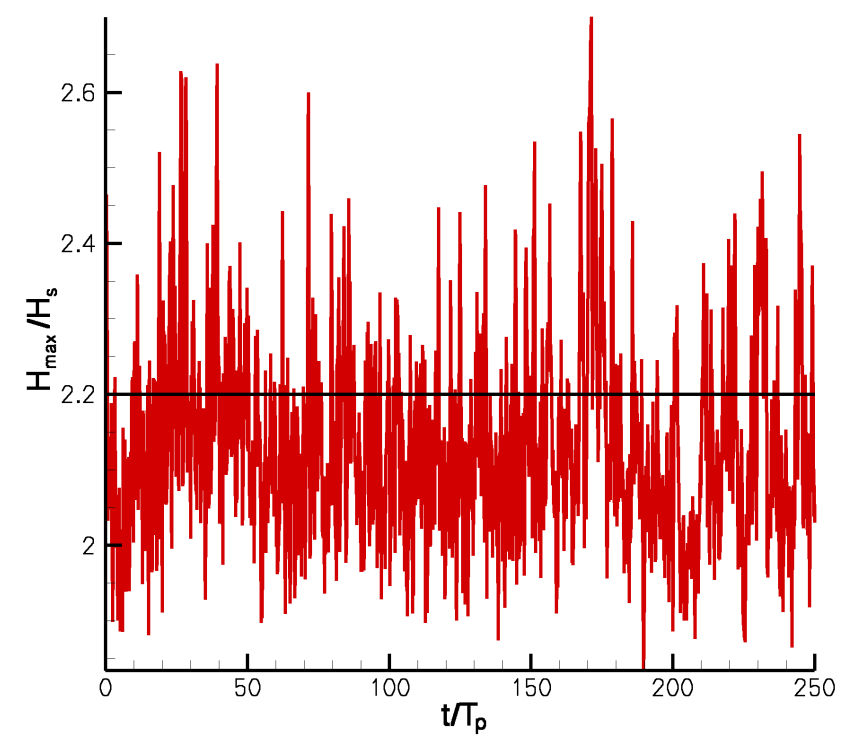

Fig. 11. Evolution of $H_{\max } / H_{S}$ during time of simulation, directionality $n=2$.

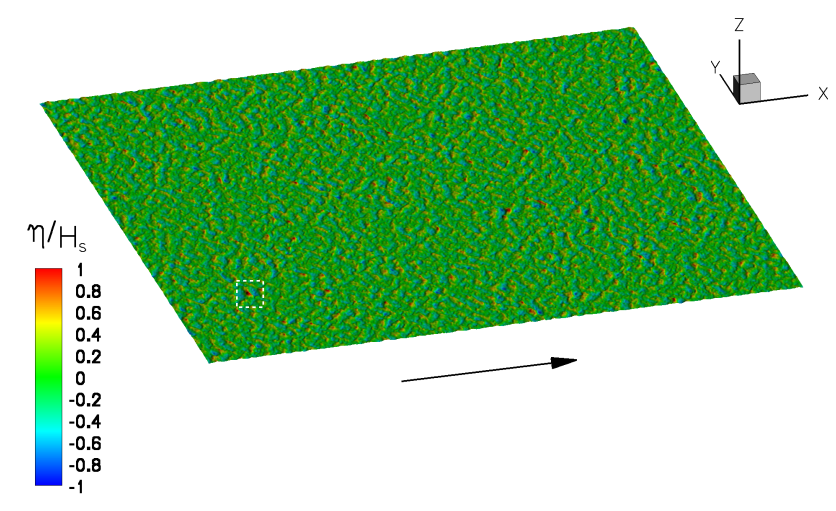

Fig. 12. 3-D surface elevation of the extreme event at $t=26.5 T_{p}$.

Appendix D). The evolution of the detection parameter $H_{\max } / H_{S}$ is plotted in Fig. 11.

At $t / T_{p}=0$, a spurious extreme event is created by the linear initialization of the simulation. The accepted threshold of 2.2 is overshot several times in this simulation. Due to the large size of the simulated domain, a high number of freak waves are observed. The large overshoot observed around $t / T_{p}=26$ lasts for several periods: it corresponds to a localized wavegroup of high amplitude that produces several freak waves in a row, as we observed previously in two dimensions.

As we carry out a three dimensional simulation, it is also interesting to look at the shape of the freak wave events. Figure 12 shows the free surface elevation at $t / T_{p}=26.5$ when the strongest event is observed. The small white square at the bottom left corner encloses the peak of which a closer view

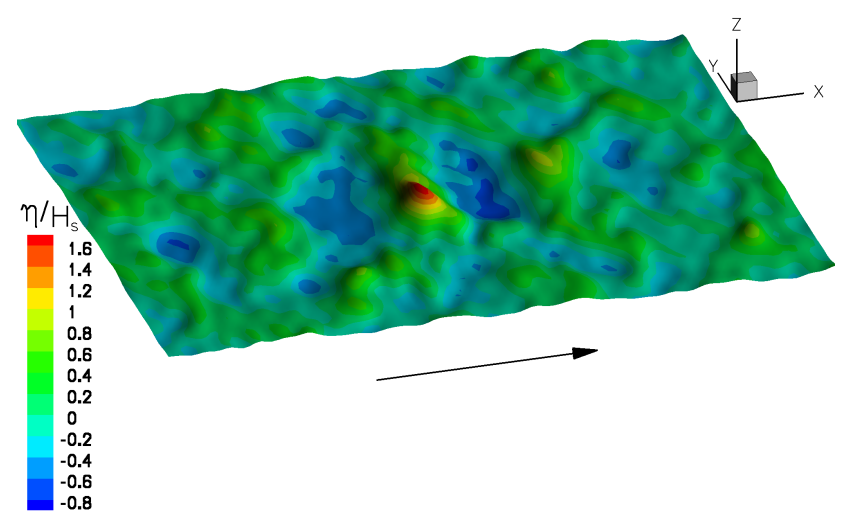

Fig. 13. Zoom on the 3-D extreme event $t=26.5 T_{p}$, $H_{\max }=2.44 H_{s}$.

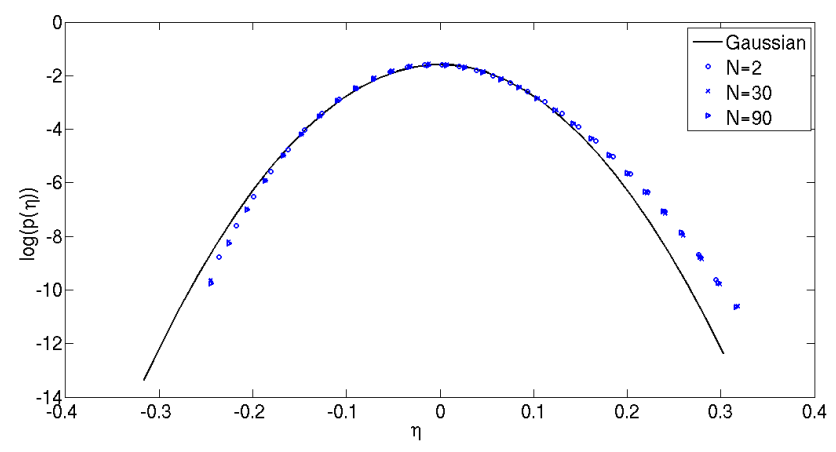

Fig. 14. Probability distribution of the free surface elevation.

is given in Fig. 13. In the main direction of propagation, this extreme event has a $1.2 \lambda_{p}$ wavelength. In the transverse direction, the wave group is rather short: the observed event is shaped like a single peak wave whose width is less than a peak wavelength. The directionality is expected to play also a significant role in modifying the probability of occurrence of extreme events.

\subsection{Influence of directionality}

Here is presented a parametric study of the influence of the directionality on freak wave formation. To this purpose, the integer $n$ defining the directional spreading of the wave spectrum is varied. The first value $n=2$ is the one used in Tanaka (2001a) and Tanaka (2001b); then more "realistic" choices of directionality are studied: $n=30$ and 90 . The first thing we looked at is the probability distribution of the free surface elevation for these different values. In Fig. 14 is shown the comparison of the probability distribution for different directional spreadings to the Gaussian one. The probability distribution is evaluated from simulations as an average over the last 20 peak periods of simulation. 


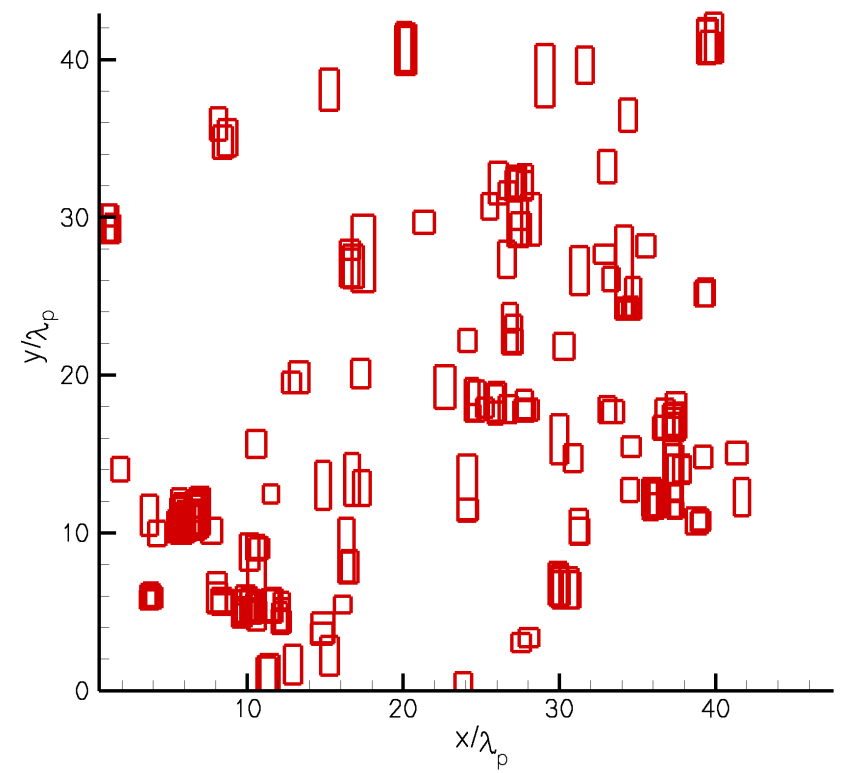

Fig. 15. Visualization of all the extreme events in the computational domain, for $n=2$.

The Fig. 14 clearly depicts the crest/trough asymmetry of the numerical wavefields: the positive elevations are more frequent than in the linear Gaussian distribution and the negative ones are less frequent. This gives a consistent indication of the nonlinear behavior of the simulated wavefields. Besides, one can observe that the directionality seems to have no direct effect on the probability distribution as the three curves for $n=2,30$ and 90 are almost superimposed.

We then focus ourselves to the occurrence and shapes of the freak events in order to characterize their possible impact on structures. All along the simulation, the position of the freak waves are recorded, as well as their shapes. The freak events are detected by a zero-crossing analysis in the mean direction of propagation which gives $H_{\max }$ and the corresponding wavelength $L_{x}$. The 2-D criterium is used $\left(H_{\max } / H_{s}>2.2\right)$. At the same time, a second zero crossing in the transverse direction gives a transverse extent $L_{y}$ and enables us to investigate the shape of the freak waves perpendicular to the direction of propagation. We report in the next three figures, Figs. 15, 16 and 17, the results for $n=2$, 30 and 90 respectively. Each rectangular box represents a detected freak wave, with the dimension of the box being the values of $L_{x}$ and $L_{y}$ characterizing the spatial extent of the freak wave.

An analysis of Fig. 15 gives an estimation of the mean transverse wavelength $L_{y_{n=2}} \simeq 2.1 \lambda_{p}$. For such a large directional spreading $(n=2)$, the freak wave events appear to have a rather short transverse extent and would look like pyramidal events (see also Fig. 13). This figure also enables us to monitor whether the freak waves appear as isolated events

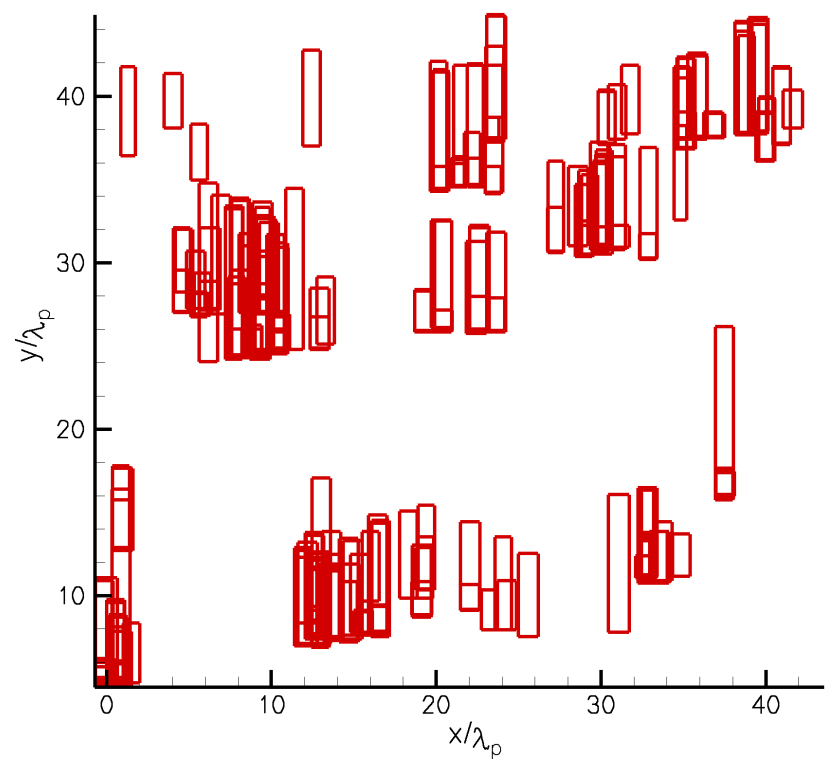

Fig. 16. Visualization of all the extreme events in the computational domain, for $n=30$.

or belong to groups of freak waves. For instance, it appears that close to the position $\left(x / \lambda_{p}, y / \lambda_{p}\right)=(6,10)$ a high amplitude wave group appears, that remains coherent for several periods in a row. This can be compared to the more isolated events also present, whose "life time" is shorter (with respect to the $H_{\max } / H_{s}$ criterion).

When we simulate a wavefield with lower spreading $n=30$, we notice in Fig. 16 that, as expected, the mean transverse wavelength is enlarged: $L_{y_{n=30}} \simeq 4.3 \lambda_{p}$. We still observe in this figure the distinction between isolated events, at $\left(x / \lambda_{p}, y / \lambda_{p}\right)=(12,40)$ for instance, and groups of extreme events, e.g. $\left(x / \lambda_{p}, y / \lambda_{p}\right)=(12,10)$. Nonetheless, the former become more scarce.

For a wavefield with narrow directional spreading $(n=90)$, the mean transverse wavelength of the freak events is again increased $L_{y_{n=90}} \simeq 5.8 \lambda_{p}$, see Fig. 17. This behavior resembles the "wall of water" commonly described by sailors.

Comparing the last three figures, one can also clearly observe that when the directionality of the wavefield is increased ( $n$ lower), the number of extreme events seems larger. In Table 2 is thus reported the number of extreme events with respect to the parameter of directionality $n$. In this table is also given the mean value of $H_{\max } / H_{s}$, i.e. the averaged value of its time evolution (such as the evolution reported in Fig. 11 for $n=2$ ).

The mean value of $H_{\max } / H_{s}$ and the number of observed extreme events both decrease when the directionality parameter increases. The occurrence of extreme events is then closely linked to the directionality, even if the probability distribution of the free surface elevation does not seem be directly influenced by this spreading (cf. Fig. 14). 


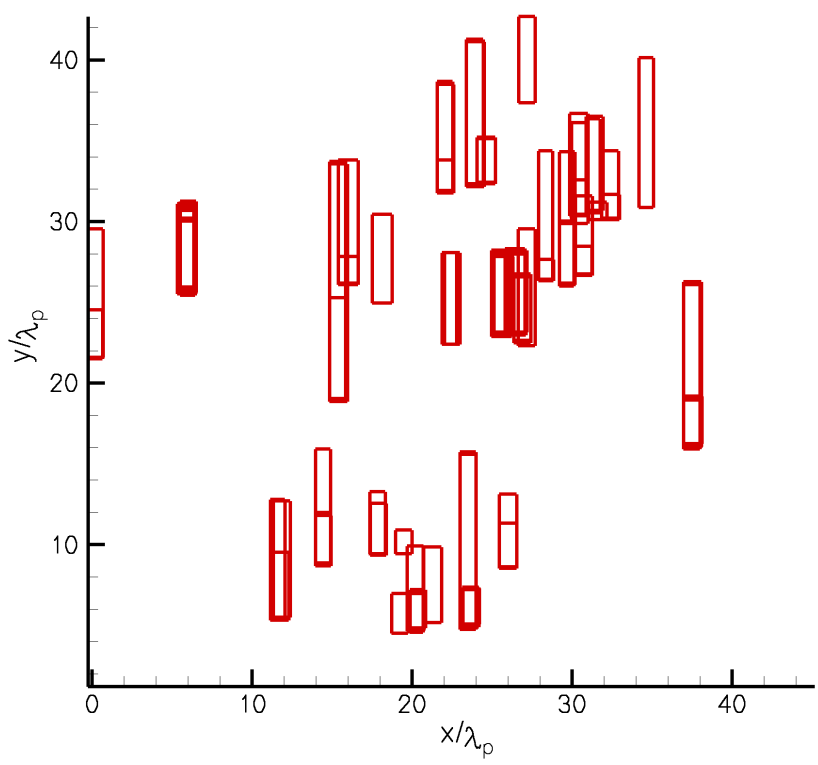

Fig. 17. Visualization of all the extreme events in the computational domain, for $n=90$.

However, our statistical analysis being made over a duration of 20 peak periods, it is likely that the population of extreme events is not representative, as too rare, during this time. The double zero-crossing analysis we performed is in a sense more appropriate to an extreme events statistics since, at each time step over the 250 peak period long simulation, each single wave is detected and the extreme ones recorded. This more convenient kind of study should be the starting point for further statistical analysis.

More systematic analysis of the detected 3-D freak waves will be made possible once the ongoing parallelization of our model is achieved. We will then be able to run more calculations in larger 3-D domains and over longer times of simulation, providing data for further statistical analyses.

\section{Conclusions}

In the present paper, a numerical investigation of extreme wave events occurring in two- and three-dimensional opensea domains has been conducted. The numerical approach used is the HOS method which has been chosen for its specific attractive features: i) a fast resolution thanks to the use of FFTs coupled to an accelerated scheme, and ii) a fast convergence providing a high accuracy when the nonlinear products involved are carefully dealiased. The enhanced model employed is presented, and successfully verified on a number of validation test cases which are briefly reported. In particular, 3-D wave-spectra simulated evolutions have been found in accordance with the Hasselmann's theory. The process followed to initialize the wavefield in the different simu-
Table 2. Number of extreme events in the simulation and mean of $H_{\max } / H_{S}$, as functions of the directionality parameter $n$.

\begin{tabular}{lccc}
\hline & $N=2$ & $N=30$ & $N=90$ \\
\hline Number of freak events & 2350 & 756 & 443 \\
Mean of $H_{\max } / H_{S}$ & 2.13 & 2.05 & 2.0 \\
\hline
\end{tabular}

lations is also described; a directional JONSWAP wave spectrum representing real sea-states is thus specified in the simulations.

Different kinds of simulations of wavefield evolutions are performed. Firstly, a forced 3-D freak wave event is generated through the directional focusing of some components of the initial sea-spectrum. This way, a very large event is obtained, whose crest height is twice the significant wave height. To get more realistic freak waves, long-time simulations of directional wave-spectrum with random initial phases have then been carried out. A 2-D evolution lasting 1000 peak periods has thus been computed, demonstrating the ability of the present model to accurately simulate series of realistic extreme events naturally appearing within the wavefield. Then, 3-D evolutions have been performed to further investigate the effect of the directionality. In the large domain of computation used (covering more than 30 square kilometers of ocean), several extreme events appear during the simulation; a group of freak waves is in particular studied. Finally, a parametric study of directionality is carried out and reveals that the number and shape of the detected freak wave events largely depends on the directional spreading. For widely spread seas, the number of extreme events is high and their width in the transverse direction tends to be limited. When the spreading decreases, the freak events number decreases and their tranverse extent increases, leading to the well known "walls of water" for quasi uni-directional seas. Another remarkable feature shown by our approach is the groupiness of freak events, especially when the directional spreading is limited, as shown in Fig. 16 where several successive freak wave events appear in the same wave group. More refined studies have to be now undertaken regarding that point.

The results obtained are hence encouraging for the pursuit of investigations in this domain using the presented HOS model. More systematic studies over repeated long-time simulations are in particular required to obtain stochastically significant results. The latter could help understanding the freak wave formation and development mechanisms, and their occurrence probability. These studies are already easily feasible in two dimensions with the present model, and will be conducted soon. And for making possible systematic threedimensional investigations, we are presently working on the parallelization of the code.

Thus, the abilities of our model have been pointed out, as well as its efficiency with huge calculations performed 
on a single processor. Nonetheless, if this model permits to simulate open-sea evolutions, it does not allow to reproduce sea evolutions obtained in wavetanks. The latter laboratory facilities are however the only possible way to experimentally study wavefield characteristics in details. Indeed, in the initial HOS formulation, no wave generation or absorption is possible, making difficult the comparison to experiments conducted in wavetanks, where are also present wall and beach reflections, generation of spurious free waves, etc. To overcome this limitation of the original HOS method, significant efforts have been dedicated to the development and validation of a new fully-spectral model HOST (for HOS Tank) able to numerically reproduce a wavetank (see Bonnefoy et al., 2004; Ducrozet et al., 2006). The next step will thus be, from open-sea extreme events such as the ones obtained in the present work, to reproduce them in the $50 \mathrm{~m} \times 30 \mathrm{~m} \times 30 \mathrm{~m}$ École Centrale de Nantes wavetank by using HOST. The key point in this process will be to adjust the wavemaker motion characteristics, in order to obtain the best fit between the target freak wave previously obtained in open-sea conditions, and its reproduction in laboratory conditions. The proposed combination of open-sea and wavetank simulations is intended to provide useful information on the freak-wave formation mechanisms.

\section{Appendix A}

\section{HOS method}

Here is described the HOS method first introduced by West et al. (1987) and Dommermuth and Yue (1987). The evaluation process of the vertical velocity at the exact free surface position $\left(W=\left.\frac{\partial \phi}{\partial z}\right|_{z=\eta(\mathbf{x}, t)}\right)$ is first based on a series expansion of the velocity potential. This series expansion with respect to $\epsilon$, being a measure of the wave steepness, gives

$\phi(\mathbf{x}, z, t)=\sum_{m=1}^{M} \phi^{(m)}(\mathbf{x}, z, t)$

where $M$ is the order of nonlinearity and $\phi^{(m)}$ a quantity of order $O\left(\epsilon^{m}\right)$. Reporting this series into the definition of the surface velocity potential $\phi^{s}=\left.\phi\right|_{z=\eta(\mathbf{x}, t)}$, we then perform a Taylor expansion of the potential $\phi$ about $z=0$. Arranging according to the order $m$ one obtains

$$
\begin{aligned}
\phi^{(1)}(\mathbf{x}, 0, t) & =\phi^{s}(\mathbf{x}, t) \\
\phi^{(2)}(\mathbf{x}, 0, t) & =-\eta \frac{\partial \phi^{(1)}}{\partial z}(\mathbf{x}, 0, t) \\
\vdots & =\quad \vdots \\
\phi^{(m)}(\mathbf{x}, 0, t) & =-\sum_{k=1}^{m-1} \frac{\eta^{k}}{k !} \frac{\partial^{k} \phi^{(m-k)}}{\partial z^{k}}(\mathbf{x}, 0, t)
\end{aligned}
$$

The full Dirichlet problem for $\phi(\mathbf{x}, z, t)$ on $z=\eta(\mathbf{x}, t)$ can then be simplified into $M$ simpler Dirichlet problems on $z=0$, respectively for $\phi^{(m)}(\mathbf{x}, z, t)$. Afterwards, the single problems are successively solved by means of a spectral method using the spectral basis functions described in Sect. 2.2.

The vertical velocity is then evaluated using the same Taylor expansion as the one previously described

$$
\begin{aligned}
W^{(1)}(\mathbf{x}, 0, t) & =\frac{\partial \phi^{(1)}}{\partial z}(\mathbf{x}, 0, t) \\
W^{(2)}(\mathbf{x}, 0, t) & =\frac{\partial \phi^{(2)}}{\partial z}(\mathbf{x}, 0, t)-\eta \frac{\partial^{2} \phi^{(1)}}{\partial z^{2}}(\mathbf{x}, 0, t) \\
\vdots & =\quad \vdots \\
W^{(m)}(\mathbf{x}, 0, t) & =-\sum_{k=0}^{m-1} \frac{\eta^{k}}{k !} \frac{\partial^{k+1} \phi^{(m-k)}}{\partial z^{k+1}}(\mathbf{x}, 0, t)
\end{aligned}
$$

The two formulations of Dommermuth and Yue (1987) and West et al. (1987) are equivalent up to this point. However, we employ the one of West et al. which propose a consistent treatment (with respect to $\epsilon$ ) of $W$ in the free surface boundary conditions (see Tanaka (2001a)). That is to say, if we denote by $W_{M}$ the sum $\sum_{m=1}^{M} W^{(m)}$ and $W_{M-2}$ the sum $\sum_{m=1}^{M-2} W^{(m)}$, Eq. (3) writes

$$
\left(1+|\nabla \eta|^{2}\right) W=W_{M}+|\nabla \eta|^{2} W_{M-2}
$$

Additional details can be found in e.g. Tanaka (2001a), Le Touzé (2003) or Bonnefoy et al. (2004).

\section{Appendix B}

\section{Acceleration procedure}

The acceleration procedure is based on the scheme presented by Fructus et al. (2005). It appears that the system of Eqs. (2, 3 ) to solve is very stiff for high frequencies, which leads to very small time steps. The key point of this scheme is, thanks to an adequate change of variables, to solve the nonlinear part of the equations as a problem in itself and not as an adjustment of the linear solution. The linear part of the equations is then analytically integrated while nonlinear evolution of the system is computed numerically using a classical fourthorder Runge-Kutta scheme with adaptative time step. For the details of the procedure, one can refer to see Fructus et al. (2005).

\section{Appendix C}

\section{Dealiasing}

The nonlinear products involved in the free surface boundary conditions $(2,3)$ are computed in the physical space instead of the spectral space. This leads to the well-known aliasing phenomenon which has to be addressed to obtain precise results (see e.g. Canuto et al., 1987). In general, dealiased 
computations can be obtained by using spectra extended with zero padding.

The number of collocation points in the physical space is then to be adequately determined for removing aliasing errors made on multiple products. The latter products are found in the free surface boundary conditions on the one hand, and within the iterative HOS obtention of $W$ (see Appendix A) on the other hand. They are $M$-products at the most (products involving $M$ terms). Applying the half rule, the number of points to use in the physical space $\left(N_{x_{d}}, N_{y_{d}}\right)$ to get a full dealiasing is

$N_{x_{d}}=\frac{M+1}{2} N_{x}, \quad N_{y_{d}}=\frac{M+1}{2} N_{y}$

However, for high-order nonlinearities, the rising of computational effort and memory allocations could become prohibitive in 3-D computations. Thus, we have chosen to perform this full dealiasing in 2-D computations and a partial dealiasing for the 3-D computations with $M>3$. We introduce the partial dealiasing as an iterative dealiasing of order $M^{\prime}<M$. Then, to compute the nonlinear products of order $M$, we perform a dealiasing on the $M^{\prime}$ first products, then on the following $M^{\prime}$, etc. and finally on the $M-k M^{\prime}$ products left. This permits for large 3-D computations to keep reasonable CPU times and memory requirements as well as a good accuracy (a detailed investigation of the accuracy of this partial dealiasing technique has been achieved and will be reported soon in a separate publication).

\section{Appendix D}

\section{Location of the freak wave events}

Classically, the so-called "freak wave" events are defined as large waves which heights exceed the significant wave height $H_{S}$ by a factor in the range [2-2.2] (see Kharif and Pelinovsky, 2003). The first (linear) approximation for representing a wavefield is that it can be considered as a stationary random normal (i.e. Gaussian) process. Under this assumption, a mathematical definition of a freak wave event can be expressed by $H_{\max }>2 H_{s}, H_{\max }$ being the height of this extreme event (see e.g. Kharif and Pelinovsky, 2003, for details).

Accounting for nonlinearities in the process, a refined definition tends to rise the limit of the freak wave height to $H_{\max }>2.2 H_{s}$. The latter limit of $2.2 H_{s}$ is now commonly accepted, for instance in the recent Rogue Waves Workshop (2005), a number of papers used this limit for defining a freak wave (Schober, 2003; Lavrenov and Porubov, 2003, see e.g.).

Once this criterion has been defined, one has to determine the height of the waves formed within the wavefield evolution. The way we analyze 2-D wavefields is to use a classical zero up-and-down crossing. We make several outputs of the surface elevation (typically about 5 outputs per wave spec- trum peak period) and perform this zero up-and-down crossing analysis of the obtained free surfaces. The significant wave height, $H_{S}$, of the wavefield as well as the maximum wave height at a given time step, $H_{\max }$, are then derived.

For analyzing 3-D wavefields, we chose to define the wave height as its height in the mean direction of propagation. Thus, we perform the same kind of analysis than in 2-D (zero up-and-down crossing along the $\mathrm{x}$-axis which is the mean direction of propagation of our wavefield) but recursively with respect to the y-direction to get the height at the wave peak location in $y$. This seemed to be the more natural way to analyze the 3-D wavefield.

Edited by: E. Pelinovsky

Reviewed by: two referees

\section{References}

Annenkov, S. and Shrira, V.: Numerical modelling of water-wave evolution based on the Zakharov equation, J. Fluid Mech., 449, 341-371, 2001.

Bonnefoy, F.: Modélisation expérimentale et numérique des états de mer complexes, Ph.D. thesis, Ecole Centrale Nantes, (in French), 2005.

Bonnefoy, F., Le Touzé, D., and Ferrant, P.: Generation of FullyNonlinear Prescribed Wave Fields using a High-Order Spectral Model, in: Proc. 14th Int. Offshore and Polar Engng. Conf., Toulon, France, 2004.

Brandini, C.: Nonlinear Interaction Processes in Extreme Waves Dynamics, Ph.D. thesis, Univ. of Firenze, 2001.

Brandini, C. and Grilli, S.: Modeling of freak wave generation in a 3D-NWT., in: Proc. 11th Int. Symp. on Offshore and Polar Engng., Vol. III, pp. 124-131, Stavanger, Norway, 2001.

Canuto, C., Haussaini, M., Quarteroni, A., and Zang, T.: Spectral Methods in Fluid Dynamics, Springer Series in Computational Physics, Springer-Verlag, Berlin, 1987.

Craig, W. and Sulem, C.: Numerical simulation of gravity waves, J. Comp. Phys., 108, 73-83, 1993.

Dommermuth, D.: The initialization of nonlinear waves using an adjustment scheme, Wave Motion, 32, 307-317, 2000.

Dommermuth, D. and Yue, D.: A high-order spectral method for the study of nonlinear gravity waves, J. Fluid Mech., 184, 267-288, 1987.

Ducrozet, G., Bonnefoy, F., Le Touzé, D., and Ferrant, P.: Implementation and Validation of Nonlinear Wave Maker Models in a HOS Numerical Wave Tank, Int. J. Offshore Polar Engng., 16, 161-167, 2006.

Dysthe, K. B.: Note on a modification to the nonlinear Schrödinger equation for application to deep water waves, R. Soc. Lond. A, 369, 105-114, 1979.

Fructus, D., Clamond, D., Grue, J., and Kristiansen, O.: An efficient model for three-dimensional surface wave simulations. Part I: Free space problems, J. Comp. Phys., 205, 665-685, 2005.

Hasselmann, K.: On the non-linear energy transfer in a gravitywave spectrum. Part 1. General theory, J. Fluid Mech., 12, 481$500,1962$. 
Haver, S., Eik, K., and Meling, T.: On the prediction of wave crest height extremes., Tech. rep., Statoil, 2002.

Kharif, C. and Pelinovsky, E.: Physical mechanisms of the rogue wave phenomenon, Eur. J. Mech. B/Fluids, 22, 603-634, 2003.

Krasitskii, V.: On reduced Hamiltonian equations in the nonlinear theory of water surface waves, J. Fluid Mech., 272, 1-20, 1994.

Lavrenov, I. and Porubov, A.: Three reasons for freak wave generation in the non-uniform current, Eur. J. Mech. B/Fluids, 22, 574-585, 2003.

Longuet-Higgins, M.: Resonant interactions between two trains of gravity waves, J. Fluid Mech., 12, 321-332, 1962.

Phillips, O.: On the dynamics of unsteady gravity waves of finite amplitude. Part 1. The elementary interactions, J. Fluid Mech., 9, 193-217, 1960.

Schäffer, H.: On the Dirichlet-Neumann Operator for Nonlinear Water Waves, in: Proc. 20th Int. Workshop on Water Waves and Floating Bodies, Longyearbayen, Norway, 2005.

Schober, C.: Melnikov analysis and inverse spectral analysis of rogue waves in deep water, Eur. J. Mech. B/Fluids, 22, 602-620, 2003.

Socquet-Juglard, H.: Spectral evolution and probability distributions of surface ocean gravity waves and extreme waves, Ph.D. thesis, University of Bergen, available at https://bora.uib.no/ handle/1956/1103, 2005.

Stiassnie, M. and Shemer, L.: On modifications of the Zakharov equation for surface gravity waves., J. Fluid Mech., 143, 47-67, 1984.
Tanaka, M.: A method of studying nonlinear random field of surface gravity waves by direct numerical simulation, Fluid Dyn. Res., 28, 41-60, 2001a.

Tanaka, M.: Verification of Hasselmann's energy transfer among surface gravity waves by direct numerical simulations of primitive equations, J. Fluid Mech., 444, 199-221, 2001 b.

Trulsen, K.: Simulating the spatial evolution of a measured time series of a freak wave, in: Proc. Rogue Waves 2000, pp. 265 273, Brest, France, 2000.

Trulsen, K. and Dysthe, K. B.: A modified nonlinear Schrödinger equation for broader bandwidth gravity waves on deep water, Wave Motion, 24, 281-289, 1996.

Le Touzé, D.: Méthodes spectrales pour la modélisation nonlinéaire d'écoulements à surface libre instationnaires, Ph.D. thesis, Ecole Centrale Nantes (in French), 2003.

Vijfvinkel, E.: Focused wave groups on deep and shallow water, Master's thesis, University of Groningen, The Netherlands, 1996.

West, B., Brueckner, K., Janda, R., Milder, M., and Milton, R.: A new numerical method for surface hydrodynamics, J. Geophys. Res., 92, 11 803-11 824, 1987.

Zakharov, V.: Stability of periodic waves of finite amplitude on the surface of a deep fluid, J. Appl. Mech. Tech. Phys., 9, 190-194, 1968. 